\title{
Acute gastrointestinal illness in adults in Germany: a population-based telephone survey
}

\author{
H. WILKING ${ }^{1}$, H. SPITZNAGEL ${ }^{1}$, D. WERBER ${ }^{1}$, C. LANGE ${ }^{2}$, A. JANSEN JA $^{1,3}$ \\ AND K. STARK ${ }^{1}$ \\ ${ }^{1}$ Department for Infectious Disease Epidemiology, Robert Koch Institute, Berlin, Germany \\ ${ }^{2}$ Department of Epidemiology and Health Reporting, Robert Koch Institute, Berlin, Germany \\ ${ }^{3}$ Scientific Advice Coordination Section, European Centre for Disease Prevention and Control, Stockholm, \\ Sweden
}

Received 4 June 2012; Final revision 18 December 2012; Accepted 6 January 2013; first published online 1 February 2013

\section{SUMMARY}

Population-based estimates of incidence and risk factors for acute gastrointestinal illness (AGI) are important for infectious disease surveillance and healthcare planning. We conducted a nationwide representative cross-sectional telephone survey of 21262 adults over a 12-month period during 2008-2009 in Germany. Participants were asked if they had either AGI-related diarrhoea or vomiting in a 4-week recall period. We estimated 0.95 episodes/person per year (95\% confidence interval 0.90-0.99), corresponding to 64.9 million episodes of AGI annually in adults, which results in 24.5 million outpatient visits, 19.9 million hospital days and 63.2 million days of work lost. We observed an overall declining trend of AGI with increasing age. Diarrhoea was more often reported than vomiting. The mean duration of illness was 3.8 days and did not differ between age groups. Social factors seemed to be weak predictors compared to state of health and health behaviour characteristics. This study allows international comparisons and contributes to the estimation of the global burden of AGI.

Key words: Estimating, gastrointestinal infections, infectious disease, prevalence of disease, virology (human) and epidemiology, zoonotic foodborne diseases.

\section{INTRODUCTION}

Acute gastrointestinal illness (AGI) has a huge public health impact in terms of disease incidence, prevention issues and healthcare costs. The majority of AGI is caused by infectious agents.

Over the last years the burden of AGI has been a field of research in many countries. The methodology for community surveys has improved over the years

\footnotetext{
* Author for correspondence: H. Wilking, DVM, M.Sc., Robert Koch Institute, Department for Infectious Disease Epidemiology, Gastrointestinal Infections, Zoonoses and Tropical Infections, DGZ-Ring 1, 13086 Berlin, Germany.

(Email: WilkingH@rki.de)
}

and a common case definition was established to ensure international comparability [1-3]. These efforts resulted in cross-sectional studies for estimates for the burden of AGI from various countries [4-18] and additionally a few population-based cohort studies [19-21].

In Germany, epidemiological analyses of infectious diseases often rely on data from the national notifiable infectious disease surveillance system. Laboratoryconfirmed cases are notified to the local public health office. Clearly, these cases represent only the tip of the surveillance pyramid [3]. Many infections go unnoticed because not all patients seek medical care. Moreover, although medically indicated, a stool 
sample is not investigated from all patients for the causative agent, which is prerequisite for notification. Varying degrees of under-ascertainment by age group, sex, socioeconomic status and geographical region can result in biased comparisons of incidence estimates. Despite the fact that many of the gastrointestinal pathogens are notifiable, there is no syndromic surveillance of AGI in Germany. In this situation conducting cross-sectional surveys using a standardized syndromic case definition for AGI is a necessary alternative. These surveys provide representative population estimates of the true burden of acute gastrointestinal disease. A cross-sectional study in North Germany in 2004 verified that in a large proportion of AGI patients an infectious agent could have been detected, indicating that the majority of these are caused by infectious agents [22]. This is why cross-sectional data on the incidence of AGI can complement the system of notifiable disease surveillance while helping to unravel true differences of disease burden from underascertainment and reporting artefacts. Furthermore, data on baseline incidence can help to interpret the data of syndromic surveillance implemented on an ad hoc basis (e.g. in the case of large and widely dispersed outbreaks) [23]. Additionally incidence estimates may help to formulate precise case definitions differentiating between baseline disease incidence and outbreak case excess during outbreak analyses of gastrointestinal diseases [24-26]. Information on determinants of socioeconomic status, personal health behaviour and self-perceived health status might help to formulate hypotheses on risk factors for related diseases. The data on healthcare utilization of AGI cases is useful for cost-effective analysis of specific interventions.

The main objective of this study was to estimate the incidence of self-reported AGI in the German adult population and investigate sociodemographic and epidemiological factors as determinants of AGI. Furthermore, we also assessed clinical manifestations and utilization of medical services in cases of AGI.

\section{METHODS}

\section{Survey methodology}

The population-based telephone survey GEDA [Gesundheit in Deutschland Aktuell (Current Health in Germany)] is part of the German health monitoring programme. The GEDA methodology has been described in detail elsewhere $[27,28]$. The sampling population consisted of the resident German-speaking adult population living in private households with a fixed telephone line (landline). The proportion of households having landline access was $89 \%$ in 2009 . Landlines are more prevalent in households with two or more persons and single households of persons aged $>60$ years [29]. Landline access is slightly more prevalent in rural villages and more prevalent in the elderly. The targeted number of respondents was 21000. The telephone-number sample was created using the Gabler-Häder design [30]; it was based on phone numbers taken from public telephone directories. In order to include people with unlisted numbers, random numbers were produced based on German area codes, thereby allowing selection of numbers not registered in directories. Altogether, a number pool consisting of both published and unpublished phone numbers was created. In order to give each element of the population the same theoretical likelihood of being interviewed, an additional selection of target persons was conducted at the household level, using the 'last-birthday method'. The computerassisted telephone interview (CATI) method was applied. Interviews were conducted by 205 interviewers in 385 working shifts which were planned to be equally distributed over the study period. To allow the maximum number of people to be contacted the shifts were worked Mondays to Fridays between 16:00 and 20:00 hours and on Saturdays from 14:00 to 18:00 hours. Interviewers were balanced regarding age and sex to avoid interviewer bias. Selected telephone numbers were attempted to be contacted up to 15 attempts. If telephone contact was made with the household, the interviewer determined if the household contained two or more adults and then asked to speak with the adult household member with the most recent birthday. Study participants were enrolled from July 2008 to June 2009, the response was $29 \cdot 1 \%$ and showed little variation by place and time. In order to improve representativeness, survey weights were generated to adjust for deviation of the target population to the German adult population based on estimates of the German Federal Statistical Office of Germany for 2009. This included a design weighting to (i) number of telephone numbers in the household and (ii) number of persons in the household, and additionally a weighting poststratification to (iii) age, (iv) sex, (v) region and (vi) education (standard classification: ISCED) [27, 28]. Unless otherwise stated, all statistical analyses account for the weights. 


\section{Case definition}

Our case definition was based on that proposed by International Collaboration on Enteric Disease 'Burden of Illness' Studies [2]. It deviates from this as vomiting was defined as having had at least three episodes. Diarrhoea was defined as $\geqslant 3$ loose stools in a 24-h period. Persons who reported having diarrhoea but then reported having fewer than three loose stools in a 24-h period were considered not to have had diarrhoea. Vomiting was defined as having had at least three episodes on one day. We defined cases of AGI as respondents who either self-reported diarrhoea $(n=1.501)$ or vomiting $(n=379)$ in the 4 weeks preceding the interview. All others were defined as non-cases.

We excluded all subjects from the analysis who had chronic gastrointestinal diseases, i.e. Crohn's disease $(n=60)$, ulcerative colitis $(n=68)$, stomach cancer and intestinal tumours $(n=31)$, irritable bowel $(n=$ $138)$ or coeliac disease $(n=22)$ or who were pregnant $(n=147)$. We excluded missing values regarding chronic gastrointestinal diseases $(n=28)$. Due to data privacy exclusion criteria for alcohol and drugs, related diarrhoea or vomiting could not be included in the study's case definition.

\section{Data analysis}

Four-week incidence $\left(I_{4 \mathrm{wk}}\right)$ as incidence proportion (expressed in \%) was calculated as

$I_{4 \mathrm{wk}}=\frac{\sum w_{k} x_{k}}{\sum w_{k}}$

where $x_{k}$ is a binary variable indicating whether a person $k$ was a case or not and where $w_{k}$ is the weight of $x_{k} .95 \%$ confidence intervals (CIs) were used as interval estimates. For reasons corresponding to other studies the annual incidence $\left(I_{\text {annual }}\right)$ was calculated as $I_{\text {annual }}=I_{4 \mathrm{wk}} \times(365 / 28)$ and expressed in terms of episodes/person per year. Odds ratios (ORs) as measures of the association between disease determinants as explanatory variables and the defined AGI cases as outcome variable were obtained using logistic regression controlled for age, sex and age $\times$ sex interaction. For categorical variables the category with the highest number of participants was chosen as reference but never one of the two extreme categories. Spatial reference is the administrative system of districts in Germany and time reference is the day of the telephone interview. For comparison of the proportion between age groups and sex, the two-tailed
$P$ value for the $z$ test from logistic regression was used. For comparison of average means between age groups and sex, the two-tailed $P$ value from linear regression was used. The analyses were performed using Stata v. 12 (Stata Corp., USA). All statistical tests and regression analyses account for the study weights using the 'svy' command in Stata v. 12.0 [31].

\section{RESULTS}

The response rate was $29 \cdot 1 \%$ and a total of 21262 interviewees responded to the survey, of which 20800 were eligible for case definition. Of these 11761 $(58 \cdot 2 \%)$ were female. Median age was 46 years (range 18-100, interquartile range: 35-60 years). Altogether $1562(7 \cdot 5 \%)$ persons reported an AGI. Incorporating the study weights the $I_{4 \mathrm{wk}}$ of AGI in adults was $7 \cdot 3 \%(95 \%$ CI $6 \cdot 9-7 \cdot 8)$ corresponding to an $I_{\text {annual }}$ of 0.95 episodes/person per year $(95 \% \mathrm{CI}$ 0.90-0.99). Extrapolated to the 2009 overall adult population of 68.3 million this resulted in an estimated $64 \cdot 9$ (95\% CI 62.0-67.8) million episodes of AGI per year in adults in Germany.

\section{Effects of age, gender, seasonality, and geographical region}

Statistically significant differences of $I_{\text {annual }}$ were found between age groups and a borderline significant age $\times$ sex interaction $(P=0 \cdot 055) \quad$ (Fig. 1). Incidence was highest in young males $\left(I_{\text {annual }}=1.29\right.$ episodes $/$ person per year, $I_{4 \mathrm{wk}}=9 \cdot 9 \%$ ), and lowest in older males $\left(I_{\text {annual }}=0.54\right.$ episodes $/$ person per year, $I_{4 \mathrm{wk}}=$ $4 \cdot 2 \%)$. We observed an overall declining trend of AGI with increasing age both in males $(P<0 \cdot 001)$ and females $(P=0.003)$. Overall, female adults had a higher odds of reporting AGI than males, but this did not reach statistical significance $(P=0 \cdot 081)$. However, in the older age groups incidence was higher in females than in males (most pronounced in the 50-59 years age group). Incidence in the Eastern German federal states (former German Democratic Republic plus West Berlin) was slightly lower $\left(I_{\text {annual }}=0.86\right.$ episodes/person per year $)$ but this difference in comparison with Western states failed to achieve significance $(P=0 \cdot 169)$. The variation of AGI during the survey period is shown in Figure 2. Incidence ranged from 0.79 in May 2008 to 1.17 in February 2009. A peak was observed from January 2009 to March 2009. 


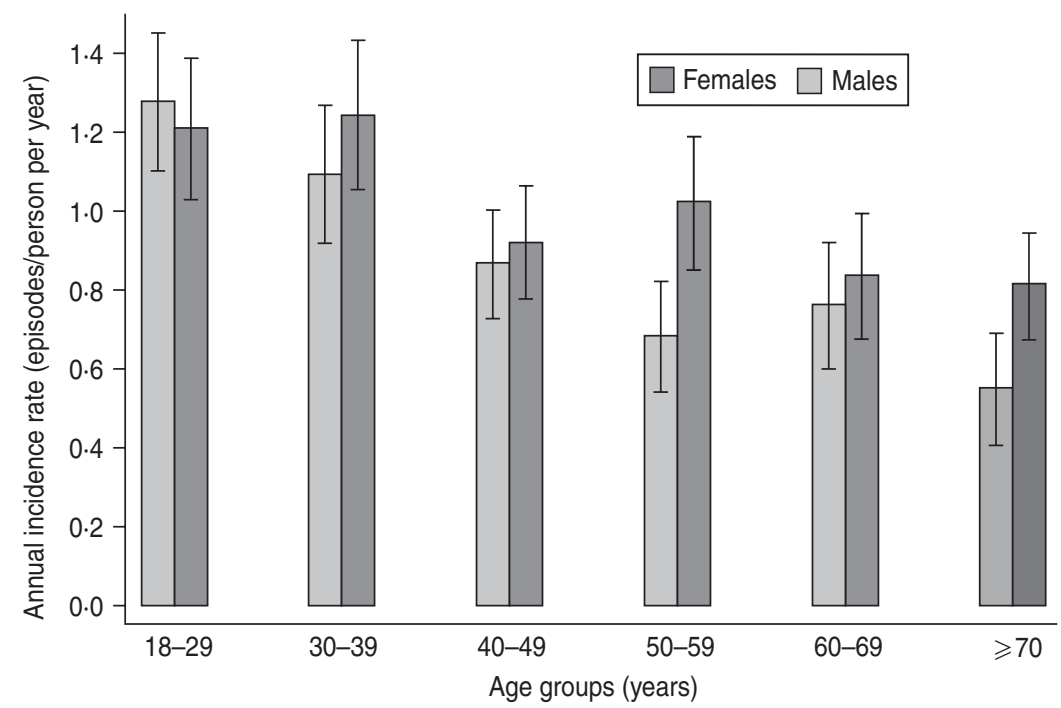

Fig. 1. Distribution of annual incidence of acute gastrointestinal illness in Germany in 2008-2009 by age and sex $(n=20800)$.

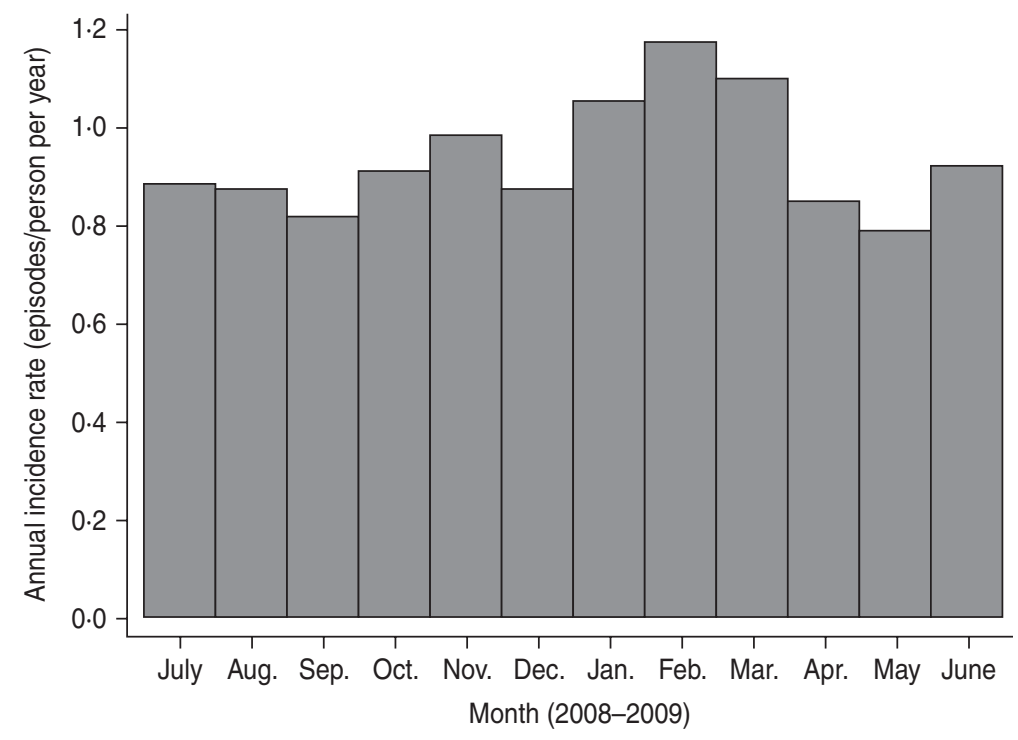

Fig. 2. Timely distribution of annual incidence of acute gastrointestinal illness by month in Germany $(n=20800)$. Time reference is the day of telephone interview.

\section{Other risk factors}

Due to the influence of age and sex of the respondents on the incidence and the odds ratios, all estimates for risk factors were adjusted for these factors and their interaction (Table 1). Generally, social characteristics seemed to be weak predictors of AGI in this study. Migrant status or migration background was not associated with AGI. Compared to a two-person household as a reference category, people living alone were more likely to report AGI (OR $1 \cdot 29$, 95\% CI $1 \cdot 07-$ $1 \cdot 55$ ), whereas larger households of four or more people (OR 0.96, 95\% CI $0 \cdot 79-1 \cdot 17$ ) or the number of infants in the household (OR 1.12, 95\% CI 0.97 $1 \cdot 29)$ were not a risk factor.

The overall health and health behaviour were significantly associated with AGI. Based on a selfreported scale from 1 to 5 (where 1 is very good and 5 is very bad), the respondents were asked for their general state of health. An association of AGI and perceived poor general health status was apparent. The question regarding personal health awareness revealed that in comparison to respondents who strongly cared for their health, people with 
Table 1. Incidence estimates and determinants for acute gastrointestinal illness (AGI) in adults in Germany, 2009 (weighted) $(n=20800)$

\begin{tabular}{|c|c|c|c|c|c|c|c|}
\hline $\begin{array}{l}\text { Determinants } \\
(n=\text { unweighted sample size) }\end{array}$ & $\begin{array}{l}\text { No. of } \\
\text { AGI cases } \\
\text { (unweighted) }\end{array}$ & $\begin{array}{l}\text { 4-week } \\
\text { incidence }\end{array}$ & $\begin{array}{l}\text { Annual } \\
\text { incidence }\end{array}$ & $(95 \% \mathrm{CI})$ & OR & $(95 \% \mathrm{CI})$ & $P$ value \\
\hline Female* $(n=11761)$ & 915 & $7 \cdot 66$ & $0 \cdot 99$ & $(0 \cdot 93-1 \cdot 06)$ & $1 \cdot 13$ & $(0 \cdot 99-1 \cdot 29)$ & $0 \cdot 081$ \\
\hline Male $(n=9039)$ & 647 & $6 \cdot 85$ & $0 \cdot 89$ & $(0.83-0.96)$ & - & - & - \\
\hline $\begin{array}{l}\text { Age (interaction with sex) } \dagger \\
\text { Female } \\
\text { Male }\end{array}$ & & - & - & - & $\begin{array}{l}0.99 \\
0.98\end{array}$ & $\begin{array}{l}(0 \cdot 99-1 \cdot 00) \\
(0 \cdot 98-0 \cdot 99)\end{array}$ & $\begin{array}{r}0 \cdot 003 \\
<0 \cdot 001\end{array}$ \\
\hline $\begin{array}{l}\text { Social determinants } \\
\text { Provenancet } \$\end{array}$ & & & & & & & \\
\hline $\begin{array}{l}\text { East German }(n=4001) \\
\text { West German }(n=16799)\end{array}$ & $\begin{array}{r}278 \\
1284\end{array}$ & $\begin{array}{l}6 \cdot 63 \\
7 \cdot 43\end{array}$ & $\begin{array}{l}0 \cdot 86 \\
0 \cdot 97\end{array}$ & $\begin{array}{l}(0 \cdot 77-0 \cdot 96) \\
(0 \cdot 92-1 \cdot 02)\end{array}$ & $\begin{array}{l}0 \cdot 89 \\
\text { Ref. }\end{array}$ & $\begin{array}{l}(0 \cdot 75-1 \cdot 05) \\
\text { Ref. }\end{array}$ & $\begin{array}{l}0 \cdot 169 \\
\text { Ref. }\end{array}$ \\
\hline $\begin{array}{l}\text { Migrant background } \$ \\
\text { No }(n=17741) \\
\text { One-sided }(n=782) \\
\text { Two-sided }(n=2275)\end{array}$ & $\begin{array}{r}1327 \\
72 \\
163\end{array}$ & $\begin{array}{l}7 \cdot 16 \\
9 \cdot 24 \\
7 \cdot 43\end{array}$ & $\begin{array}{l}0 \cdot 93 \\
1 \cdot 20 \\
0 \cdot 97\end{array}$ & $\begin{array}{l}(0 \cdot 88-0 \cdot 98) \\
(0 \cdot 92-1 \cdot 49) \\
(0 \cdot 84-1 \cdot 10)\end{array}$ & $\begin{array}{l}\text { Ref. } \\
1 \cdot 26 \\
0.95\end{array}$ & $\begin{array}{l}\text { Ref. } \\
(0 \cdot 93-1 \cdot 72) \\
(0 \cdot 75-1 \cdot 20)\end{array}$ & $\begin{array}{l}\text { Ref. } \\
0 \cdot 138 \\
0 \cdot 649\end{array}$ \\
\hline $\begin{array}{l}\text { Net household income }(€):\|\| \\
\quad<1500(n=3622) \\
1500-2125(n=2914) \\
2126-2750(n=2996) \\
2751-3750(n=3075) \\
>3750(n=2992)\end{array}$ & $\begin{array}{l}326 \\
224 \\
200 \\
226 \\
207\end{array}$ & $\begin{array}{l}7 \cdot 99 \\
7 \cdot 20 \\
6 \cdot 86 \\
7 \cdot 71 \\
7 \cdot 17\end{array}$ & $\begin{array}{l}1 \cdot 04 \\
0 \cdot 94 \\
0 \cdot 89 \\
1 \cdot 00 \\
0 \cdot 93\end{array}$ & $\begin{array}{l}(0 \cdot 92-1 \cdot 15) \\
(0 \cdot 82-1 \cdot 06) \\
(0 \cdot 78-1 \cdot 01) \\
(0 \cdot 88-1 \cdot 13) \\
(0 \cdot 80-1 \cdot 07)\end{array}$ & $\begin{array}{l}1 \cdot 15 \\
1 \cdot 02 \\
0 \cdot 90 \\
\text { Ref. } \\
0 \cdot 92\end{array}$ & $\begin{array}{l}(0 \cdot 92-1 \cdot 44) \\
(0 \cdot 80-1 \cdot 30) \\
(0 \cdot 70-1 \cdot 15) \\
\text { Ref. } \\
(0 \cdot 72-1 \cdot 16)\end{array}$ & $\begin{array}{l}0 \cdot 206 \\
0 \cdot 898 \\
0 \cdot 395 \\
\text { Ref. } \\
0 \cdot 471\end{array}$ \\
\hline $\begin{array}{l}\text { No. of persons in household: } \\
1(n=4730) \\
2(n=7421) \\
3(n=3829) \\
\geqslant 4(n=5245)\end{array}$ & $\begin{array}{l}390 \\
483 \\
304 \\
384\end{array}$ & $\begin{array}{l}8 \cdot 00 \\
6 \cdot 30 \\
8 \cdot 11 \\
7 \cdot 56\end{array}$ & $\begin{array}{l}1 \cdot 04 \\
0 \cdot 82 \\
1 \cdot 05 \\
0 \cdot 99\end{array}$ & $\begin{array}{l}(0 \cdot 92-1 \cdot 16) \\
(0 \cdot 75-0 \cdot 89) \\
(0 \cdot 95-1 \cdot 16) \\
(0 \cdot 89-1 \cdot 08)\end{array}$ & $\begin{array}{l}1 \cdot 29 \\
\text { Ref. } \\
1 \cdot 09 \\
0 \cdot 96\end{array}$ & $\begin{array}{l}(1 \cdot 07-1 \cdot 55) \\
\text { Ref. } \\
(0 \cdot 89-1 \cdot 33) \\
(0 \cdot 79-1 \cdot 17)\end{array}$ & $\begin{array}{l}\mathbf{0} \cdot 007 \\
\text { Ref. } \\
0 \cdot 405 \\
0 \cdot 703\end{array}$ \\
\hline $\begin{array}{l}\text { Health and health behaviour dete } \\
\text { Care for health } t\end{array}$ & & & & & & & \\
\hline $\begin{array}{l}\text { Very strong }(n=2684) \\
\text { Strong }(n=8738) \\
\text { Moderate }(n=7722) \\
\text { Less strong }(n=1256) \\
\text { Not at all }(n=362)\end{array}$ & $\begin{array}{r}187 \\
567 \\
622 \\
147 \\
32\end{array}$ & $\begin{array}{l}6 \cdot 82 \\
6 \cdot 06 \\
7 \cdot 90 \\
10 \cdot 99 \\
10 \cdot 07\end{array}$ & $\begin{array}{l}0 \cdot 89 \\
0 \cdot 79 \\
1 \cdot 03 \\
1 \cdot 42 \\
1 \cdot 31\end{array}$ & $\begin{array}{l}(0 \cdot 76-1 \cdot 01) \\
(0 \cdot 72-0 \cdot 86) \\
(0 \cdot 95-1 \cdot 11) \\
(1 \cdot 20-1 \cdot 65) \\
(0 \cdot 95-1 \cdot 68)\end{array}$ & $\begin{array}{l}1 \cdot 18 \\
\text { Ref. } \\
1 \cdot 30 \\
1 \cdot 85 \\
1 \cdot 70\end{array}$ & $\begin{array}{l}(0 \cdot 94-1 \cdot 47) \\
\text { Ref. } \\
(1 \cdot 12-1 \cdot 52) \\
(1 \cdot 44-2 \cdot 37) \\
(1 \cdot 06-2 \cdot 75)\end{array}$ & $\begin{array}{l}0 \cdot 158 \\
\text { Ref. } \\
\mathbf{0} \cdot \mathbf{0 0 1} \\
<\mathbf{0} \cdot \mathbf{0 0 1} \\
\mathbf{0} \cdot \mathbf{0 2 9}\end{array}$ \\
\hline $\begin{array}{l}\text { General state of health }: \\
\text { Very good }(n=5071) \\
\text { Good }(n=10613) \\
\text { Moderate }(n=4024) \\
\text { Bad }(n=841) \\
\text { Very bad }(n=219)\end{array}$ & $\begin{array}{r}214 \\
723 \\
459 \\
125 \\
38\end{array}$ & $\begin{array}{l}4 \cdot 00 \\
6 \cdot 52 \\
10 \cdot 38 \\
12 \cdot 45 \\
14 \cdot 22\end{array}$ & $\begin{array}{l}0 \cdot 52 \\
0 \cdot 85 \\
1 \cdot 35 \\
1 \cdot 62 \\
1 \cdot 85\end{array}$ & $\begin{array}{l}(0 \cdot 45-0 \cdot 60) \\
0 \cdot 79-0 \cdot 91 \\
1 \cdot 24-1 \cdot 46 \\
1 \cdot 36-1 \cdot 88 \\
1 \cdot 32-2 \cdot 39\end{array}$ & $\begin{array}{l}0 \cdot 52 \\
\text { Ref. } \\
2 \cdot 12 \\
2 \cdot 83 \\
3 \cdot 28\end{array}$ & $\begin{array}{l}(0 \cdot 43-0 \cdot 63) \\
\text { Ref. } \\
1 \cdot 79-2 \cdot 51 \\
2 \cdot 16-3 \cdot 72 \\
2 \cdot 08-5 \cdot 18\end{array}$ & $\begin{array}{l}<0.001 \\
\text { Ref. } \\
<0.001 \\
<0.001 \\
<0.001\end{array}$ \\
\hline $\begin{array}{l}\text { Consumption of fruits: } \\
\text { Daily }(n=13040) \\
\text { Less }(n=7745)\end{array}$ & $\begin{array}{l}892 \\
668\end{array}$ & $\begin{array}{l}6 \cdot 62 \\
8 \cdot 29\end{array}$ & $\begin{array}{l}0 \cdot 86 \\
1 \cdot 08\end{array}$ & $\begin{array}{l}0 \cdot 81-0 \cdot 92 \\
1 \cdot 00-1 \cdot 16\end{array}$ & $\begin{array}{l}0 \cdot 82 \\
\text { Ref. }\end{array}$ & $\begin{array}{l}0 \cdot 72-0 \cdot 95 \\
\text { Ref. }\end{array}$ & $\begin{array}{l}\mathbf{0} \cdot \mathbf{0 1 0} \\
\text { Ref. }\end{array}$ \\
\hline $\begin{array}{l}\text { Consumption of vegetables: } \\
\text { Daily }(n=10151) \\
\text { Less }(n=10638)\end{array}$ & $\begin{array}{l}770 \\
792\end{array}$ & $\begin{array}{l}7 \cdot 19 \\
7 \cdot 33\end{array}$ & $\begin{array}{l}0 \cdot 94 \\
0 \cdot 95\end{array}$ & $\begin{array}{l}0 \cdot 87-1 \cdot 00 \\
0 \cdot 89-1 \cdot 02\end{array}$ & $\begin{array}{l}0 \cdot 96 \\
\text { Ref. }\end{array}$ & $\begin{array}{l}0 \cdot 84-1 \cdot 10 \\
\text { Ref. }\end{array}$ & $\begin{array}{l}0 \cdot 578 \\
\text { Ref. }\end{array}$ \\
\hline $\begin{array}{l}\text { Consumption of fruit juicet } \\
\text { Daily }(n=3915) \\
\text { Less }(n=16840)\end{array}$ & $\begin{array}{r}274 \\
1286\end{array}$ & $\begin{array}{l}6 \cdot 61 \\
7 \cdot 42\end{array}$ & $\begin{array}{l}0 \cdot 86 \\
0 \cdot 96\end{array}$ & $\begin{array}{l}0 \cdot 76-0 \cdot 96 \\
0 \cdot 92-1 \cdot 02\end{array}$ & $\begin{array}{l}0 \cdot 91 \\
\text { Ref. }\end{array}$ & $\begin{array}{l}0 \cdot 76-1 \cdot 08 \\
\text { Ref. }\end{array}$ & $\begin{array}{l}0 \cdot 276 \\
\text { Ref. }\end{array}$ \\
\hline $\begin{array}{l}\text { Alcohol consumption } \$ \\
\text { Never }(n=3220) \\
\leqslant 1 \text { time a month }(n=4773) \\
2-4 \text { times a month }(n=6234) \\
2-3 \text { times a week }(n=4183) \\
\geqslant 4 \text { times a week }(n=2346)\end{array}$ & $\begin{array}{l}303 \\
364 \\
405 \\
280 \\
205\end{array}$ & $\begin{array}{l}9 \cdot 09 \\
7 \cdot 61 \\
6 \cdot 04 \\
6 \cdot 14 \\
8 \cdot 33\end{array}$ & $\begin{array}{l}1 \cdot 18 \\
0 \cdot 99 \\
0 \cdot 79 \\
0 \cdot 80 \\
1 \cdot 08\end{array}$ & $\begin{array}{l}1 \cdot 06-1 \cdot 30 \\
0 \cdot 89-1 \cdot 09 \\
0 \cdot 71-0 \cdot 87 \\
0 \cdot 70-0 \cdot 90 \\
0 \cdot 94-1 \cdot 23\end{array}$ & $\begin{array}{l}1 \cdot 72 \\
1 \cdot 31 \\
\text { Ref. } \\
1 \cdot 14 \\
1.83\end{array}$ & $\begin{array}{l}1 \cdot 41-2 \cdot 10 \\
1 \cdot 09-1.59 \\
\text { Ref. } \\
0.93-1.39 \\
1.44-2.33\end{array}$ & $\begin{array}{c}<\mathbf{0 . 0 0 1} \\
\mathbf{0 . 0 0 5} \\
\text { Ref. } \\
0 \cdot 200 \\
<0.001\end{array}$ \\
\hline
\end{tabular}


Table 1 (cont.)

\begin{tabular}{|c|c|c|c|c|c|c|c|}
\hline $\begin{array}{l}\text { Determinants } \\
(n=\text { unweighted sample size })\end{array}$ & $\begin{array}{l}\text { No. of } \\
\text { AGI cases } \\
\text { (unweighted) }\end{array}$ & $\begin{array}{l}\text { 4-week } \\
\text { incidence }\end{array}$ & $\begin{array}{l}\text { Annual } \\
\text { incidence }\end{array}$ & $(95 \% \mathrm{CI})$ & OR & $(95 \% \mathrm{CI})$ & $P$ value \\
\hline \multicolumn{8}{|l|}{ Body mass index $(\mathrm{BMI}) \uparrow$} \\
\hline Underweight (BMI <18.5) $(n=459)$ & 41 & $8 \cdot 60$ & $1 \cdot 12$ & $0 \cdot 76-1 \cdot 48$ & $1 \cdot 20$ & $0 \cdot 79-1>84$ & $0 \cdot 396$ \\
\hline Normal weight $(n=10584)$ & 694 & $6 \cdot 44$ & $0 \cdot 84$ & $0 \cdot 77-0 \cdot 90$ & Ref. & Ref. & Ref. \\
\hline Overweight (BMI 25-30) $(n=7005)$ & 504 & $7 \cdot 01$ & $0 \cdot 91$ & $0.84-0.99$ & $1 \cdot 30$ & $1 \cdot 10-1 \cdot 52$ & $0 \cdot 002$ \\
\hline Obesity $(\mathrm{BMI}>30)(n=2790)$ & 283 & $9 \cdot 57$ & $1 \cdot 25$ & $1 \cdot 11-1 \cdot 38$ & $1 \cdot 87$ & $1 \cdot 54-2 \cdot 27$ & $<0 \cdot 001$ \\
\hline \multicolumn{8}{|l|}{ Diabetest } \\
\hline Yes $(n=1215)$ & 116 & $9 \cdot 67$ & $1 \cdot 26$ & $1 \cdot 07-1 \cdot 45$ & $1 \cdot 81$ & $1 \cdot 38-2 \cdot 38$ & $<0.001$ \\
\hline No $(n=19585)$ & 1446 & $7 \cdot 07$ & $0 \cdot 92$ & $0 \cdot 87-0.97$ & Ref. & Ref. & Ref. \\
\hline Total $(n=20800)$ & 1562 & $7 \cdot 26$ & 0.95 & $0.90-0.99$ & - & - & - \\
\hline
\end{tabular}

OR, Odds ratio; CI, confidence interval.

$*$ OR adjusted for age.

$\dagger$ In years and reference category 18 years.

$\$$ Adjusted for age, sex, and age $\times$ sex interaction.

$\S$ Whole of Berlin as East Germany.

I A person was defined as having a one-sided migrant background if at least one of the parents was not born in Germany, a two-sided migrant background if the person had no German citizenship, moved to Germany after birth or both parents were not born in Germany.

\|I In Euro (€), discretized by quintiles.

Bold values indicate significance.

self-reported moderate, poor or very poor personal health awareness more frequently reported AGI. However, respondents who self-reported a very strong care did not benefit. Daily consumption of fruits was inversely associated with AGI (OR 0.82, CI $0.72-0.95$ ), whereas daily consumption of vegetables and fruit juice were not statistically related for AGI.

Alcohol consumption had a two-way association with risk for AGI. Overall, 6234/20756 (30\%) of the interviewees reported drinking alcohol 2-4 times a month. People who reported drinking $\geqslant 4$ times a week had a significantly higher incidence (OR 1.83 , 95\% CI 1.44-2.33). By contrast, respondents who reported drinking less than the reference category or who reported never drinking alcohol (OR 1.72, 95\% CI 1.41-2.10) were also significantly more at risk. In an analysis of the influence of alcohol consumption stratified on diarrhoea and vomiting, the results are qualitatively the same for AGI as the combined outcome.

The body mass index (BMI) was a significant predictor of AGI. With each increase of BMI score the risk of AGI increased by $5 \%$. The risk for obese persons $(\mathrm{BMI}>30)$ was the highest. Diabetes mellitus was reported by $5.8 \%$ of the participants and is significantly associated with AGI in our study (OR 1.81, 95\% CI 1·38-2.38).

\section{Symptoms, severity and healthcare utilization}

Of the 1562 respondents who met the case definition for AGI, $78.0 \%$ reported diarrhoea, $11.9 \%$ reported vomiting and $10 \cdot 1 \%$ experienced both symptoms (Table 2). Bloody diarrhoea was reported by $3 \cdot 6 \%$. Mean duration of illness was 3.7 days without significant differences by age and sex.

More than one third (37.8\%) of cases sought outpatient medical care and $3.4 \%$ were hospitalized. Overall, $13 \cdot 8 \%$ of the cases reported providing a stool sample for microbiological examination. This was significantly more often reported by the elderly (24.4\% in those aged $>70$ years) and by females $(16 \cdot 3 \%)$. There were no significant differences between West Germany and East Germany regarding the proportion of patients reporting an outpatient visit $(P=0.49)$ and providing a stool sample $(P=0 \cdot 70)$. Altogether $49 \cdot 8 \%$ of the AGI cases reported having taken medication against AGI, $31 \cdot 2 \%$ reported a medical prescription and $10 \cdot 6 \%$ of all AGI cases reported antibiotic therapy. The latter was significantly more often prescribed for elderly patients. Fever was associated with antibiotic therapy prescriptions $(P<0 \cdot 001)$; however, bloody diarrhoea was not $(P=0 \cdot 462)$. In our study, $23 \cdot 2 \%$ of the AGI patients had to stay away from work with a mean duration of work absenteeism of $4 \cdot 2$ days. 
Table 2. Proportions and average means for associated factors and medical actions taken of cases of acute gastrointestinal illness by age and sex ( $n=1562)$

\begin{tabular}{|c|c|c|c|c|c|c|c|c|c|c|c|}
\hline & \multicolumn{7}{|c|}{ Age group (years) } & \multirow{2}{*}{$\begin{array}{l}P \text { value } \\
\text { for age* }\end{array}$} & \multirow[b]{2}{*}{ Female } & \multirow[b]{2}{*}{ Male } & \multirow{2}{*}{$\begin{array}{l}P \text { value } \\
\text { for sex }\end{array}$} \\
\hline & Total & $18-29$ & $30-39$ & $40-49$ & $50-59$ & $60-69$ & $\geqslant 70$ & & & & \\
\hline Diarrhoea $(\%)$ & $88 \cdot 1$ & $81 \cdot 0$ & $87 \cdot 6$ & $87 \cdot 7$ & $92 \cdot 3$ & $91 \cdot 5$ & $94 \cdot 8$ & $<0 \cdot 001$ & $87 \cdot 8$ & $88 \cdot 7$ & 0.671 \\
\hline Vomiting ( $\geqslant 3$ times/day) $(\%)$ & $22 \cdot 0$ & $30 \cdot 4$ & $28 \cdot 2$ & $20 \cdot 0$ & $16 \cdot 1$ & $14 \cdot 4$ & $14 \cdot 4$ & $<0 \cdot 001$ & $23 \cdot 6$ & $20 \cdot 1$ & $0 \cdot 214$ \\
\hline Bloody diarrhoea $(\%)$ & $3 \cdot 6$ & $3 \cdot 8$ & $4 \cdot 3$ & $3 \cdot 9$ & $3 \cdot 3$ & $3 \cdot 6$ & $3 \cdot 3$ & 0.904 & $3 \cdot 3$ & $4 \cdot 0$ & $0 \cdot 598$ \\
\hline Fever $\left(>38 \cdot 5^{\circ} \mathrm{C}\right)(\%)$ & $10 \cdot 0$ & $16 \cdot 4$ & $11 \cdot 8$ & $10 \cdot 7$ & $5 \cdot 5$ & $4 \cdot 5$ & $6 \cdot 3$ & $0 \cdot 001$ & $10 \cdot 4$ & $9 \cdot 9$ & $0 \cdot 823$ \\
\hline Stool sample $(\%)$ & $13 \cdot 8$ & $7 \cdot 7$ & $8 \cdot 6$ & $12 \cdot 4$ & $16 \cdot 0$ & $22 \cdot 5$ & $24 \cdot 4$ & $<0.001$ & $16 \cdot 3$ & $10 \cdot 9$ & $0 \cdot 033$ \\
\hline Travel related $(\%)$ & $6 \cdot 6$ & $7 \cdot 6$ & $7 \cdot 5$ & $9 \cdot 5$ & $4 \cdot 1$ & $6 \cdot 1$ & $2 \cdot 7$ & 0.028 & $4 \cdot 6$ & $9 \cdot 0$ & $0 \cdot 002$ \\
\hline Outpatients $(\%) \dagger$ & $37 \cdot 8$ & $38 \cdot 2$ & $30 \cdot 2$ & $31 \cdot 9$ & $36 \cdot 0$ & $42 \cdot 9$ & $55 \cdot 5$ & $0 \cdot 002$ & $37 \cdot 7$ & $38 \cdot 0$ & 0.939 \\
\hline Hospitalized $(\%)$ & $3 \cdot 4$ & $0 \cdot 9$ & $4 \cdot 5$ & $1 \cdot 5$ & $3 \cdot 2$ & $5 \cdot 5$ & $7 \cdot 6$ & $0 \cdot 026$ & $3 \cdot 8$ & $3 \cdot 0$ & $0 \cdot 557$ \\
\hline Work absenteeism (\%) & $23 \cdot 2$ & $33 \cdot 2$ & $32 \cdot 2$ & $28 \cdot 6$ & $20 \cdot 7$ & n.a. & n.a. & n.a. & $20 \cdot 4$ & $26 \cdot 5$ & n.a. \\
\hline Medication taken $(\%)$ & $49 \cdot 8$ & $54 \cdot 2$ & $40 \cdot 9$ & $45 \cdot 9$ & $51 \cdot 4$ & $52 \cdot 3$ & $57 \cdot 3$ & $0 \cdot 247$ & $52 \cdot 4$ & $46 \cdot 7$ & 0.085 \\
\hline Medication prescribed (\%) & $31 \cdot 2$ & $30 \cdot 4$ & $22 \cdot 9$ & $27 \cdot 9$ & $30 \cdot 2$ & $40 \cdot 4$ & $43 \cdot 9$ & $0 \cdot 002$ & $30 \cdot 6$ & $31 \cdot 9$ & 0.693 \\
\hline Antibiotics prescribed $(\%)$ & $10 \cdot 6$ & $10 \cdot 0$ & $5 \cdot 6$ & $8 \cdot 1$ & $11 \cdot 4$ & $17 \cdot 3$ & $16 \cdot 0$ & $0 \cdot 011$ & $11 \cdot 3$ & $9 \cdot 8$ & $0 \cdot 517$ \\
\hline Mean number of stools per case of diarrhoea & $4 \cdot 7$ & $4 \cdot 8$ & $4 \cdot 7$ & $4 \cdot 7$ & $5 \cdot 0$ & $4 \cdot 6$ & $4 \cdot 3$ & $0 \cdot 164$ & $4 \cdot 7$ & $4 \cdot 7$ & $0 \cdot 829$ \\
\hline Mean duration of symptoms (days) & $3 \cdot 7$ & $3 \cdot 9$ & $3 \cdot 6$ & $3 \cdot 7$ & $3 \cdot 6$ & $3 \cdot 6$ & $4 \cdot 0$ & $0 \cdot 863$ & $3 \cdot 8$ & $3 \cdot 6$ & $0 \cdot 564$ \\
\hline Mean duration of hospitalisation (days) & $9 \cdot 0$ & $3 \cdot 6$ & $8 \cdot 1$ & $5 \cdot 9$ & $8 \cdot 8$ & $9 \cdot 7$ & $13 \cdot 3$ & $0 \cdot 003$ & $9 \cdot 0$ & $9 \cdot 1$ & $0 \cdot 966$ \\
\hline Mean duration of work absenteeism (days) & $4 \cdot 2$ & $3 \cdot 8$ & $3 \cdot 9$ & $5 \cdot 1$ & $4 \cdot 0$ & n.a. & n.a. & $0 \cdot 231$ & $4 \cdot 4$ & $4 \cdot 1$ & $0 \cdot 628$ \\
\hline
\end{tabular}

n.a., Not applicable.

* Proportions: two-tailed $P$ value for the $z$ test from logistic regression; means: two-tailed $P$ value from linear regression.

$\dagger$ Either doctor in private practice, medical service in hospital without hospitalization.

Bold values indicate significance. 
In an extrapolation of these proportions to the total adult German population of $68 \cdot 3$ million, the results of our survey add up to $24 \cdot 5$ million outpatient visits, $9 \cdot 0$ million stool sample analyses, nearly 6.9 million antibiotic prescriptions dispensed, 19.9 million days in hospital and 63.2 million working days lost.

\section{DISCUSSION}

This is the first survey conducted in Germany which is able to obtain nationwide representative data on the incidence and distribution of AGI in the population. The study, limited to adults, provides nationally representative estimates for disease burden. The sampling procedure and the statistical weighting ensure unbiased samples regarding age, sex, geographical region and education status. For adults young age was the strongest predictor for AGI in this study. It is possible that older persons have been repeatedly exposed to gastrointestinal pathogens during the course of life and acquired relative immunity. Alternatively risky behaviour of young adults leaving home and beginning to prepare their own food (second weaning) could be a contributing factor.

\section{International comparison}

Incidence for AGI in adults in Germany is generally in line with observations in similar studies in other countries. Comparisons between countries have to consider the varying case definitions. We used a more restrictive criterion for the identification of AGIrelated vomiting. This could explain differences to other countries, e.g. to the Danish study with a higher proportion of cases with vomiting explaining a higher overall incidence [15]. Additionally, most of the studies, unlike ours, included children and adolescents and the cut points for age groups vary between the analyses. A decreasing trend for age is reported in all similar studies. From neighbouring European Union member-state countries Denmark reported higher incidence in younger adults but the same in the elderly (from $I_{\text {annual }}=2 \cdot 0$ in the 20-29 years age group to $I_{\text {annual }}=0.75$ in those aged $\geqslant 70$ years) [15], Italy reported lower incidence in the elderly (from $I_{\text {annual }}=1 \cdot 11$ in the 10-24 years age group to $I_{\text {annual }}=0.33$ in those aged $\geqslant 75$ years) [18] and Poland almost the same incidence $\left(I_{\text {annual }}=0.9\right.$ in the 15-64 years age group) [6] as in Germany. Furthermore, reported incidence of AGI seem to be generally in the same range as in the USA [11], Canada [16],
Hong Kong [12] and Australia [10], lower than in New Zealand [4], Cuba [5] and Norway [14], respectively, but higher than in Great Britain [21], Malta [7], Ireland [17] and Malaysia [9].

Generally, similar proportions of bloody diarrhoea in cases of AGI are reported from Denmark and Canada. Higher levels of bloody diarrhoea are published from New Zealand and Australia and lower levels from studies of the USA, Ireland and Malta.

\section{Seasonality and geography}

The distribution of AGI during the 1-year study period can be explained by the seasonal variation in infections with viral enteric pathogens (most prominently norovirus). This assumption is supported by the fact that the peak of AGI in January and February, as observed in the present study, corresponds to the 2009 peak of norovirus activity in Germany in the fourth calendar week which was observed from the available surveillance systems of notifiable disease [32]. By contrast, seasonality of AGI of bacterial origin (most prominently Campylobacter and Salmonella) peaks in August but the summer season is not prominent for AGI incidence in this study. A similar seasonal AGI pattern was discovered by a study in Northern Germany who also found viral pathogens more frequently detected [22]. In neighbouring countries seasonality is similar $[6,15,18]$ while other studies report seasonal peaks in summer [10]. There is no difference in the distribution of AGI between East and West Germany which has important implications for the interpretation of surveillance data of enteric diseases in Germany. Since reunification in 1990 a substantial higher incidence in the notification of infectious diseases was observed for East Germany mainly regarding gastrointestinal pathogens $[32,33]$. This increased notification rate is not mirrored by the syndromic level in our survey results which demonstrate no differences between the two parts of the country. Based on our study, higher notification rates in the East are not explainable by different food consumption habits, differing population dynamics or the higher daycare attendance rates in infants as has been hypothesized. Different disease awareness in the population could be an alternative explanation.

\section{Determinants}

Social and economic factors are not or only weakly associated with the risk of AGI. Although a differing 
lifestyle can be assumed, migrants and people with migrant background have no increased risk for AGI. The respondent's self-reported income groups are statistically related to AGI. Thus, in contrast to developing countries, financial factors seem to have a minor influence in industrialized countries like Germany where sanitary hygiene standards and microbiological quality of food and drinking water are not (in the same degree) dependent on socioeconomic status. Larger household size is not related to disease, not even the number of infants. This is surprising, assuming that a large proportion of the AGI cases in this study became infected with pathogens via the faecaloral route related to contact frequencies with other persons [22, 32]. However, infants who are more susceptible for faecal-oral transmission were not included in the study. Single-person households with a higher infection rate are a notable exception which could be explained by frequent visits to cafeterias and purchases at fast-food outlets (e.g. takeaways).

Characteristics of health and health behaviour are prominent determinants of AGI. The degree of negative perception of an individual's own health status is linearly correlated with the incidence of AGI. This may reflect the influence of concomitant diseases on gastrointestinal infections. A similar effect could be observed regarding self-reported care for health.

There are conflicting results regarding alcohol consumption in this study. It can be hypothesized that people who frequently drink alcohol, as was asked in our study, also do so excessively and therefore report vomiting and diarrhoea. Additionally, frequent consumption of alcohol could affect the overall immunity of the participants [34, 35]. By contrast, people who never drink alcohol are also more likely to report AGI. The reasons for this could be attributed to confounding factors such as alcohol abstinence due to health grounds which also lead to AGI or the defining conditions of diarrhoea and vomiting.

Eating fruit is preventive, drinking fruit juice and eating vegetables is not. This could be a true protective effect or a proxy for nutritional habits. An increase in BMI is associated with AGI. From the probabilistic point of view, eating more in greater quantities and frequency increases the likelihood of consuming a foodborne pathogen as also does a hypothesized increased consumption of risk food. In addition to this effect, eating fat- and carbohydraterich and low-fibre diets could have a harmful effect on the gastrointestinal flora and a high BMI is correlated with a generally impaired immunity [36].

\section{Healthcare utilization}

AGI is common in adults in Germany and represents a significant burden of illness. Utilization of healthcare service is high in all age groups. Surveys from other countries that asked about prescription of antibiotics reported less utilization than in our survey in Germany $(10 \cdot 6 \%)$. This is despite the fact that we did not include children and adolescents, groups which are known to have higher prescription rates for antibiotic treatment than adults. In Ireland 5.6\% of AGI cases self-report antibiotic use [37], 8.3\% in the USA [37], $3.8 \%$ in Canada [37], $3.6 \%$ in Australia [37], 6.5\% in Italy [18] and $6 \cdot 4 \%$ in New Zealand [4]. Prescription and consumption practices appear to be considerably different in Germany. It remains to be investigated if this disproportion is caused by disease inherent factors, different diagnostic guidelines or differences in healthcare systems. The higher proportions of faecal sampling for diagnostics in the elderly and in females will presumably result in differences in notification rates of gastrointestinal pathogens. This could explain increased overall incidence of laboratory-confirmed cases of norovirus in females in Germany [32]. Incidence estimates based on notification data of viral pathogens increase in those aged $\geqslant 60$ years which was not seen in our study. In the future, differences in age and sex in the distribution of enteric pathogens should be interpreted against this background.

\section{Limitations and strengths}

Our study relies on a large number of individuals which generates precise estimates. Persons in households without a landline phone connection could not participate in the study which presumably resulted in underrepresentation of some social groups. This might have introduced selection bias; however, applying the study weights attempts to correct for basic demographic factors. Additionally, the response rate of $29 \cdot 1 \%$ indicates a possibility for selection bias. The study is limited in a way that a full assessment of all possible underlying chronic disease was not able to be performed. The international comparability of incidence estimates is hampered by different survey methodologies and cases definitions. We decided that the AGI-related vomiting criterion requires at least three episodes as we believe that a single episode of vomiting may not be specific enough for an AGI infection. This differs from other work groups. Missing 
exclusion criteria for alcohol and drugs in the cases definition were related to data privacy and considered by the authors as a minor deviation. Many gastrointestinal symptoms occur as a consequence of primary respiratory infections and this aspect could not be assessed. A future study could benefit from including respiratory symptoms as part of the survey process. The study concerned AGI from all aetiological agents and did not distinguish between bacterial or viral origin. Pathogen-specific risk estimates would provide a better insight into the risk of frequent bacterial gastroenteritis $[8,38-40]$. The telephone interview did not encompass all assumable risk factors for AGI or even precisely record dietary habits. Instead it focused on some general and partially subjective underlying factors and self-reporting of those might introduce exposure misclassification; this certainly restricts the interpretation of our study.

\section{CONCLUSION}

The burden of AGI is high in adults in Germany. Almost 9/10 individuals experience an episode each year. Risk factors are more pronounced on the general state of health and health behaviour than on the social situation. Markedly, high rates of prescribed antibiotics in AGI patients should be further investigated. The health-promoting effect of eating fruits and the prevention of obesity, diabetes and alcohol abuse should be increasingly supported in Germany. This survey should be complemented with children and adolescents in the future.

\section{ACKNOWLEDGEMENTS}

This study was partly funded by the European Community Network of Excellence, MedVetNet and the Ministry of Health in Germany. The authors thank M. Höhle for his support and comments on the statistical analyses.

\section{DECLARATION OF INTEREST}

None.

\section{REFERENCES}

1. Cantwell LB, et al. The effect of different recall periods on estimates of acute gastroenteritis in the United States, FoodNet Population Survey 2006-2007. Foodborne Pathogens and Disease 2010; 7: 1225-1228.
2. Majowicz SE, et al. A common, symptom-based case definition for gastroenteritis. Epidemiology and Infection 2008; 136: 886-894.

3. O'Brien SJ, et al. Methods for determining disease burden and calibrating national surveillance data in the United Kingdom: the second study of infectious intestinal disease in the community (IID2 study). BMC Medical Research Methodology 2010; 10: 39.

4. Adlam SB, et al. Acute gastrointestinal illness in New Zealand: a community study. Epidemiology and Infection 2011; 139: 302-308.

5. Aguiar Prieto P, et al. Burden of self-reported acute gastrointestinal illness in Cuba. Journal of Health, Population, and Nutrition 2009; 27: 345-357.

6. Baumann-Popczyk A, et al. Incidence of self-reported acute gastrointestinal infections in the community in Poland: a population-based study. Epidemiology and Infection 2012; 140: 1173-1184.

7. Gauci C, et al. The magnitude and distribution of infectious intestinal disease in Malta: a population-based study. Epidemiology and Infection 2007; 135: 12821289.

8. Gkogka E, et al. Risk-based estimate of effect of foodborne diseases on public health, Greece. Emerging Infectious Diseases 2011; 17: 1581-1590.

9. Gurpreet K, et al. Incidence and determinants of acute diarrhoea in Malaysia: a population-based study. Journal of Health, Population, and Nutrition 2011; 29: 103-112.

10. Hall GV, et al. Frequency of infectious gastrointestinal illness in Australia, 2002: regional, seasonal and demographic variation. Epidemiology and Infection 2006; 134: 111-118.

11. Herikstad H, et al. A population-based estimate of the burden of diarrhoeal illness in the United States: FoodNet, 1996-7. Epidemiology and Infection 2002; 129: 9-17.

12. Ho SC, et al. Acute gastroenteritis in Hong Kong: a population-based telephone survey. Epidemiology and Infection 2010; 138: 982-991.

13. Jones TF, et al. A population-based estimate of the substantial burden of diarrhoeal disease in the United States; FoodNet, 1996-2003. Epidemiology and Infection 2007; 135: 293-301.

14. Kuusi M, et al. Incidence of gastroenteritis in Norway a population-based survey. Epidemiology and Infection 2003; 131: 591-597.

15. Muller L, Korsgaard H, Ethelberg S. Burden of acute gastrointestinal illness in Denmark 2009: a populationbased telephone survey. Epidemiology and Infection 2012; 140: 290-298.

16. Sargeant JM, Majowicz SE, Snelgrove J. The burden of acute gastrointestinal illness in Ontario, Canada, 20052006. Epidemiology and Infection 2008; 136: 451-460.

17. Scallan E, et al. Acute gastroenteritis in northern Ireland and the Republic of Ireland: a telephone survey. Communicable Disease and Public Health 2004; 7: $61-67$.

18. Scavia G, et al. The burden of self-reported acute gastrointestinal illness in Italy: a retrospective survey, 
2008-2009. Epidemiology and Infection 2012; 140: 1193 1206.

19. Wheeler JG, et al. Study of infectious intestinal disease in England: rates in the community, presenting to general practice, and reported to national surveillance. The Infectious Intestinal Disease Study Executive. British Medical Journal 1999; 318: 1046-1050.

20. de Wit MA, et al. Sensor, a population-based cohort study on gastroenteritis in the Netherlands: incidence and etiology. American Journal of Epidemiology 2001; 154: 666-674.

21. Tam CC, et al. Longitudinal study of infectious intestinal disease in the UK (IID2 study): incidence in the community and presenting to general practice. Gut 2012; 61: 69-677.

22. Karsten C, et al. Incidence and risk factors for community-acquired acute gastroenteritis in northwest Germany in 2004. European Journal of Clinical Microbiology \& Infectious Diseases 2009; 28: 935-943.

23. Wadl M, et al. Enhanced surveillance during a large outbreak of bloody diarrhoea and haemolytic uraemic syndrome caused by Shiga toxin/verotoxin-producing Escherichia coli in Germany, May to June 2011. Eurosurveillance 2011; 16.

24. Buchholz U, et al. German outbreak of Escherichia coli O104:H4 associated with sprouts. New England Journal of Medicine 2011; 365: 1763-1770.

25. Frank C, et al. Epidemic profile of Shiga-toxinproducing Escherichia coli O104:H4 outbreak in Germany. New England Journal of Medicine 2011; 365: 1771-1780.

26. Wilking $\mathbf{H}$, et al. Identifying risk factors for shiga toxinproducing Escherichia coli by payment information. Emerging Infectious Diseases 2012; 18: 169-170.

27. Kroll LE, Lampert T. Unemployment, social support and health problems: results of the GEDA study in Germany, 2009. Deutsches Ärzteblatt international 2011; 108: 47-52.

28. Schmich P. Jentsch, Fieldwork monitoring in telephone surveys. In: Häder S, Häder $M$, Kühne $M$ (eds).
Telephone Surveys in Europe: Research and Practice. Heidelberg: Springer, 2012, pp. 295-314.

29. Eurobarometer. E-communications household survey, 2010 (http://ec.europa.eu/public_opinion/archives/ebs/ ebs_335_en.pdf). Accessed 18 December 2012.

30. Gabler S, Häder S. Telephone Sampling Surveys: Methodological Innovation and Applications [in German]. Münster/New York/München/Berlin: Waxmann Verlag, 2002.

31. Stata. Stata statistical software for professionals survey commands. College Station, Texas, USA: Stata Corporation, 2012 (http://www.stata.com/capabilities/ survey-commands/). Accessed 31 August 2012.

32. Robert-Koch-Institut. Annual Report on infectious diseases in Germany for 2009 [in German], 2010 (http:/l www.rki.de/DE/Content/Infekt/Jahrbuch/Jahrbuch_2009. pdf?_blob=publicationFile).

33. Reintjes R, et al. Infectious diseases before and after German unification: trends in mortality and morbidity. European Journal of Epidemiology 2001; 17: 1105-1110.

34. MacGregor RR. Alcohol and immune defense. Journal of the American Medical Association 1986; 256: 1474 1479 .

35. Szabo G. Consequences of alcohol consumption on host defence. Alcohol and Alcoholism 1999; 34: 830-841.

36. Milner JJ, Beck MA. The impact of obesity on the immune response to infection. Proceedings of the Nutrition Society 2012; 71: 298-306.

37. Scallan E, et al. Prevalence of diarrhoea in the community in Australia, Canada, Ireland, and the United States. International Journal of Epidemiology 2005; 34: 454- 460.

38. Ethelberg S, et al. Risk factors for diarrhea among children in an industrialized country. Epidemiology 2006; 17: 24-30.

39. Scallan E, et al. Foodborne illness acquired in the United States - unspecified agents. Emerging Infectious Diseases 2011; 17: 16-22.

40. Scallan E, et al. Foodborne illness acquired in the United States - major pathogens. Emerging Infectious Diseases 2011; 17: 7-15. 